\title{
Functional PCS power supply system with EV battery storage for stable PV power delivery
}

\author{
Kiyotaka Fuji $^{\mathrm{a}, \mathrm{b}^{*}}$, Yoshihito Shirai ${ }^{\mathrm{a}}$, Takahisa Kawagoe ${ }^{\mathrm{b}}$, Atsushi Shiota ${ }^{\mathrm{b}}$, \\ Yasunori Mitani ${ }^{\mathrm{b}}$, Yaser Soliman Qudaih ${ }^{\mathrm{b}}$ \\ ${ }^{a}$ Department of Biological Functions and Engineering Graduate School of Life Science and Systems Engineering, \\ Kyushu Institute of Technology, Kitakyushu 808-0196, Japan \\ ${ }^{b}$ Department of Electrical and Electronic Engineering, Kyushu Institute of Technology, Kitakyushu 804-8550, Japan
}

\begin{abstract}
This research proposes a residential Photovoltaic-Power Conditioning Subsystem (PV-PCS) in a functional and stable power supply system with battery storage (Electric Vehicle [EV] storage etc.) to reduce PV output fluctuation. In PV power generation, $\mathrm{PV}$ power fluctuations caused by weather changes make it difficult to obtain stable power output. Further, it can then be expected that this adversely affects the power system. In this paper, functional power supply system model is constructed with power fluctuation suppression control system using bidirectional DC/DC converter and existing residential PCS and EV battery storage. Furthermore, simulation results of the electric battery power suppression element of the PV power fluctuation are also shown by using Simple Moving Average (SMA) control method to suppress PV power fluctuation. PV power suppression system using existing residential PCS has an advantage that can not only suppress the energy change during normal operation but can also construct the isolated power supply in an emergency case of power supply loss. In this emergency case, EV battery storage control provides the power to critical loads during utility outage. This feature is not available without storage. Also, effectiveness of PV-PCS interconnection stable power supply system with existing residential PCS in Japan was proposed. Moreover, a basic research of solar power generation amount of solar radiation estimation and the resulting simulation on information acquisition method of solar energy capacity using Geographic Information System (GIS) are presented.
\end{abstract}

Keywords: Renewable energy, electric vehicles storage, photovoltaic power generation, power conditioning subsystem, $P V$ power fluctuation suppression, geographic information system

\section{Introduction}

Recently, discontinuation of the operation of nuclear power plants resulted to a tight state of power supply in Japan. For this reason, power companies in Japan must respond to the power demand and make full use of hydroelectric power and thermal power generation. To respond to this power demand, the independent power supply system capable of flexible operation must be effective. These systems should be capable to be independent from the main power system, and should be able to cover the demand and provide continuous supply of power during blackout in emergency cases once isolated and working offgrid. Electric vehicle (EV) can be regarded as a movable electric power storage device. EV power storage is also useful in absorption of power fluctuation of photovoltaic (PV) generation system installed in households. This can contribute to a low-carbon society and reduction of peak power is possible. Furthermore, spread of implementation of movable EV power storage device with PV power generation is expected not only due to its advantage in location but also to build a quick autonomous power supply system [1]-[6].

In addition, there is a global effort towards the realization of a low carbon society and reduction of $\mathrm{CO}_{2}$ emission. In Japan, along with the impact of the Great East Japan Earthquake in March 2011, there

\footnotetext{
* Manuscript received July 10, 2014; revised July 30, 2014.

Corresponding author: Kiyotaka Fuji; Tel.: +81-93-884-3243; E-mail address: fuji-k@ele.kyutech.ac.jp

doi: $10.12720 /$ sgce.4.1.45-51
} 
is a remarkable impact of the new Feed-In Tariff (FIT) scheme enforced in July 2012 for renewable energy sources. This promotes introduction of natural energy power generation such as PV power generation and Power Conditioning Subsystem (PCS). However, the variation of PV power generation greatly affects the power supply system and the output power is varied using PCS in different weather conditions. On the other hand, it is well known that increase of terminal voltage occurs due to surplus of electric power during light load. At this time, if stable power is supplied by reducing power fluctuation of the PV power generation, it is expected to contribute to the reduction of large power fluctuation suppressing device required in PV mass deployment [7]- [10].

Furthermore, a power supply independent of the power system grid in the case of blackout such as a disaster is required. Smart community demonstration of the Ministry of Economy, Trade and Industry has pointed out that smart grid deployment cost is expensive (the expansion of implementation area, for example) and that the spread of smart grid to existing city is difficult. However, by implementing Geographic Information System (GIS) which can be operated by the local government, states of existing residential PV, high accuracy estimation of power generation in PV introduction, and adaptation of the best distributed energy system can be known and achieved. Moreover, autonomous power supply system of around $1 \mathrm{~kW}$ can be constructed easily by using a combination of electric vehicle storage system, PV, and commercial DC/DC converter with DC output control function. Commercially available AC/DC inverters can be easily constructed in a free-standing system. However, these cannot be interconnected with the power grid which becomes a serious problem. This system cannot be used as an interconnected power supply system and can only be used as a stand-alone power system [11], [12].

This article is based on basic control technology analysis of the residential PV-PCS connected EV battery storage. The objective of this paper is to present the basic research of the functional power supply flexible system using EV energy storage and existing residential PCS with grid interconnection conforming to the power system guidelines. Various control studies have been done in order to suppress PV power fluctuation. In this research, PV power fluctuation suppression control using Simple Moving Average (SMA) method is used. Furthermore, by comparing the amount of solar radiation and solar PV power by solar radiation measurement based on weather variation factor, GIS effective solar radiation amount estimation is briefly discussed [13], [14].

This paper can be divided into five parts. The first part discusses brief introduction of the PV power fluctuation. The second part describes the residential PV-PCS stable power supply delivery system configuration. The third part discusses the PV power fluctuation suppression simulation. The fourth part reveals examination of solar radiation estimation method. Lastly, the fifth part is the conclusion.

\section{Residential PV-PCS Stable Power Supply Delivery System Configuration}

\subsection{PV power fluctuation}

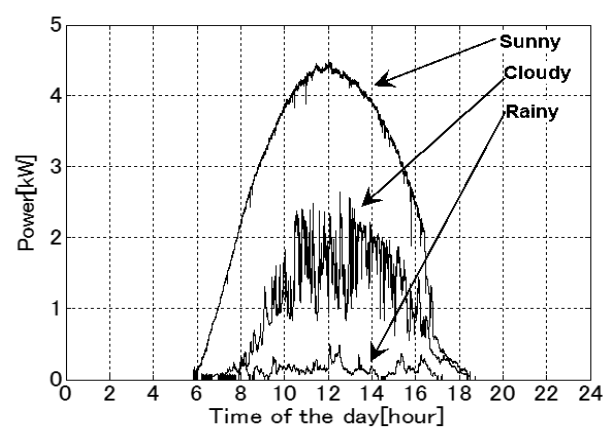

Fig. 1. PCS output data with PV power fluctuation in weather.

PV power generation greatly depends on the weather. Its output power fluctuates in weather conditions such as rainy, cloudy and sunny. Fig. 1 shows the PV generation power output of a Japan standard PCS 
system in a 3kW PV panel installed at the rooftop of Building No. 10 in Kyushu Institute of Technology (Kyutech).

In fine weather, the average power output of the PCS is about $3 \mathrm{~kW}$. In a cloudy weather, PCS output power is fluctuating heavily. PCS output power is the lowest during rainy weather.

\subsection{Residential PV-PCS standard model structure}

A Japanese Standard PCS equivalent structure is shown in Fig. 2. When this standard PCS can be utilized easily to the PV system power fluctuation, flexible power supply system is constructed as existing residential system. Photovoltaic (PV) Power conditioning Subsystem (PCS) is commercially available and product specifications are defined to comply with the guidelines of the grid interconnection. Adaptability of the circuit system according to that type is important when building a system with PCS. In Fig. 2 (a), PCS is configured to control the charging and discharging of the battery. However, there is a limit to the storage control of the PCS given the DC input voltage range. Further, in a commercially available PCS, the connection terminal of the DC voltage is not built externally. Therefore, in an existing residential PCS, construction of a grid integrated system connected to the DC smoothing part is difficult. Fig. 2 (b) shows the unit in which gradation control is performed on AC output so as to have a low ripple by monitoring the DC voltage smoothing part and through a bidirectional buck-boost circuit. Commercially available PCS are more efficient, but since control matching with the AC output part and the DC smoothing part is achieved more closely than (a), system configuration for grid interconnection between the DC part is difficult. In Fig. 2 (c), since the complicated control apparatus with RB-IGBT (Reverse Blocking - Insulated Gate Bipolar Transistor) is configured, the grid interconnection system configured to be connected to standard DC section becomes more difficult. Thus, in order to realize a power supply system that can be readily constructed, connection of a PV fluctuation suppressing device in the PV input of an existing residential PCS is effective.

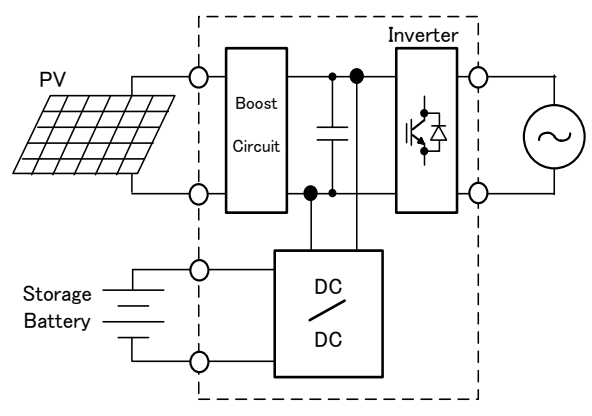

(a)

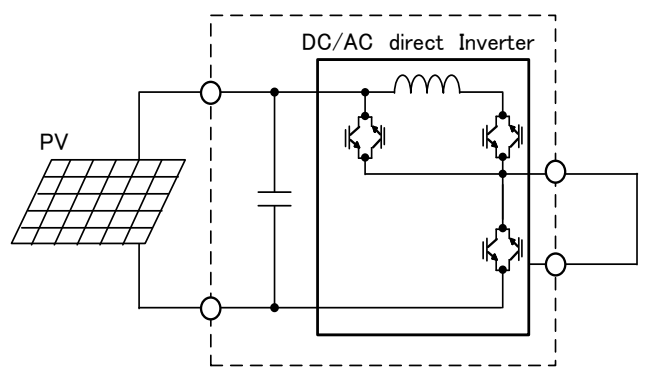

(c)

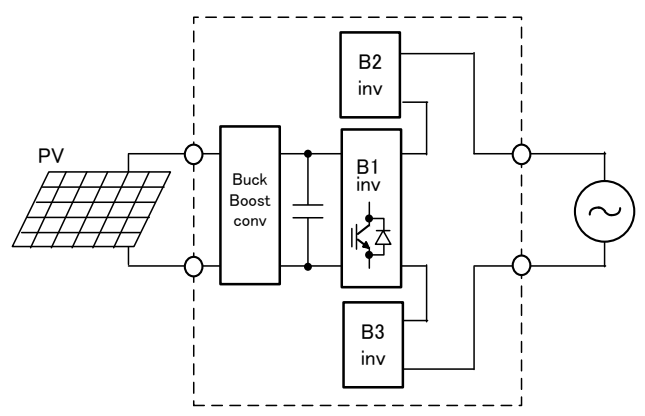

(b)

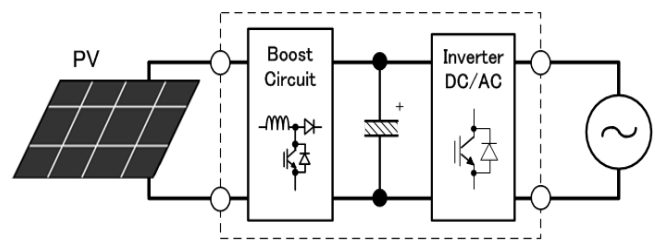

(d)

Fig. 2. Residential PV-PCS structure in Japanese model: (a) DC bus linked type, (b) Gradationally controlled type, (c) DC/AC Buck-Boost direct controlled type, (d) Standard PV-PCS type.

\subsection{PV-PCS stable power supply delivery system model}

A stable power supply delivery system model is shown in Fig. 3. A general PV system consisting of 
solar cell and power conditioner for fluctuation suppression system is implemented with DC voltage model. The system is constructed by connecting EV storage battery through bidirectional DC/DC converter to control the power. In this system, it is very important to consider the fluctuation suppression method of PV output and the required charging and discharging power in determining the specifications of bidirectional DC/DC converter.

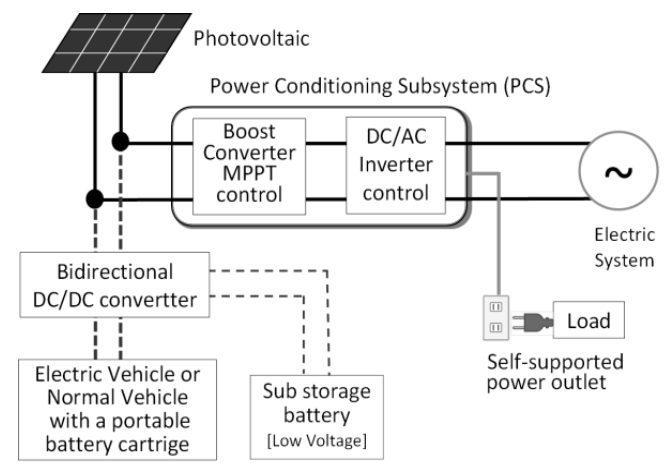

Fig. 3. Stable power supply delivery system model.

In this research, Simple Moving Average (SMA) method is used in suppressing the fluctuation of PV output. Charging and discharging power of the battery storage is determined by the difference between the actual PV output value and the target value set by the SMA method. PV output Fluctuation Suppression waveform within one day is shown in Fig. 5. The required charging and discharging power value used in determining the specifications of the bidirectional DC/DC converter was evaluated using the power data taken in one year of the $2.88 \mathrm{~kW}$ PV installed in Kyutech. In this proposed structure, one advantage is that the PV fluctuation suppression control device can be connected easily to the input of the general-purpose residential PCS.

\subsection{Simple moving average method}

PV power Fluctuation Suppression (PVFS) system can be utilized using Simple Moving Average (SMA) method for PV power Fluctuation Suppression (PVFS) such as real power control and load leveling. This paper uses PVFS in enhancing the performance of fluctuation control as it can provide active power compensation in a short period of time. This strategy can be considered as a new method. Recently, a huge number of electric companies and independent system operators have shown growing interest in PVFS due to decreasing cost of batteries.

In Japan, photovoltaic generator is promoted as the most effective system among natural power sources. However, it is well-known that electric power fluctuations occur due to change in weather conditions. Furthermore, when a large amount of PV has been introduced to the power system, electric power fluctuation may result to frequency fluctuation as well as voltage fluctuation in the power system. According to the Bureau of Statistics, the total number of houses in Japan in 2008 is approximately 57 million in which 50 million houses are occupied. As a result, stable power supply operation will be interrupted occasionally. In order to overcome these issues, the system in this research is described.

In using Simple Moving Average (SMA) method for PV generation output, PV generator Fluctuation Suppression (PVFS) control can be utilized effectively.

In the SMA method, the reference for station output at " $k$ th" control interval $\left(P_{P V F S}^{*}(k)\right)$ is set to the Simple Moving Average (sampling time is 1 [s], averaging time is $T_{M A}[\mathrm{~s}]$ ) of $P_{P V}$ as

$$
P_{P V F S}^{*}(k)=\frac{1}{T_{M A}} \sum_{t=1}^{T_{M A}} P_{P V}(k-i)
$$

Longer $T_{M A}$ results to smaller short-term fluctuation of $P_{P V F S}^{*}$. However, it also lengthens the time delay 
of $P_{P V F S}^{*}$ to $P_{P V}$. Longer time delay requires larger $P_{B A T}$. As a result, too long averaging time increases the risk of power capacity violation and deteriorates the fluctuation suppression performance. $\mathrm{PV}$ power Fluctuation Suppression (PVFS) control using SMA method is discussed in the next section.

\section{PV Power Fluctuation Suppression Simulation}

\subsection{Simulation analysis of PVFS method using SMA}

In Fig. 4 and Fig. 5, PVFS simulation block diagram and PVFS simulation waveform using SMA are shown. In PV power Fluctuation Suppression (PVFS) control, the difference between the PV power output and SMA calculation of PV power output is taken as fluctuation suppression control value for battery storage battery charge and discharge amount. In this simulation, the output power measurement data of the actual PV-PCS device is used. PV power output when implementing photovoltaic generator fluctuation suppression using SMA control within 5 minutes, 15minutes, and 30minutes are shown in Fig. 6 (a), Fig. 6 (b), and Fig. 6 (c). In the data on the left of Fig. 6, SMA control simulation output power and the output power of photovoltaic power generation are shown. In the data on the middle, assumed EV battery charge and discharge control power for PV fluctuation suppression is shown. In the data on the right figure, the total electric energy of the EV Battery electric power consumption for suppressing PV power fluctuation is shown. When SMA control time becomes larger, PV power fluctuation is suppressed more. However, the charge-discharge electric power becomes greater in the same case.



Fig. 4. PVFS simulation block diagram using SMA.

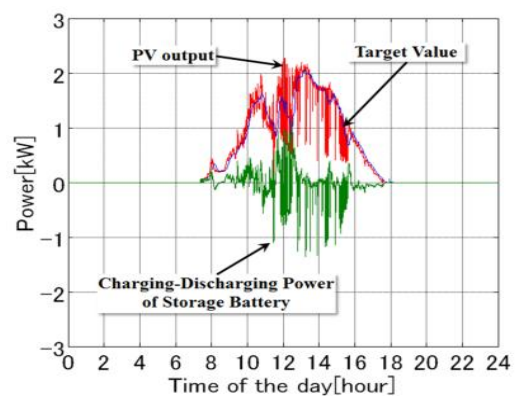

Fig. 5. PVFS simulation waveform using SMA.

(Left)PV-SMA power, (Middle) EV Battery power, (Right) EV Battery electric power consumption
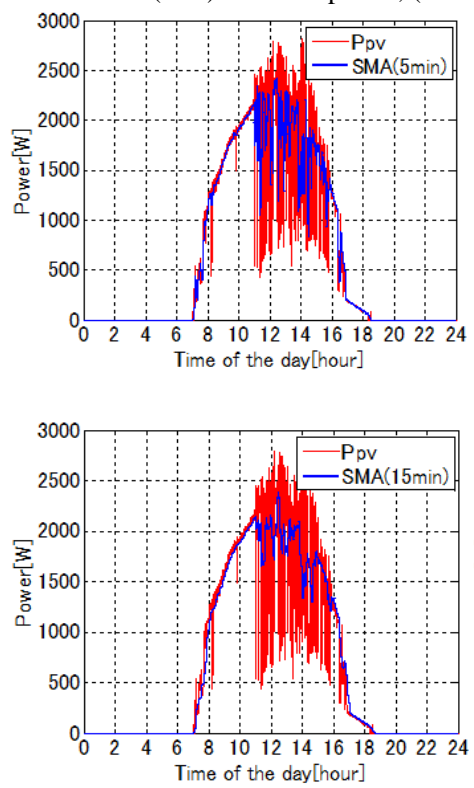

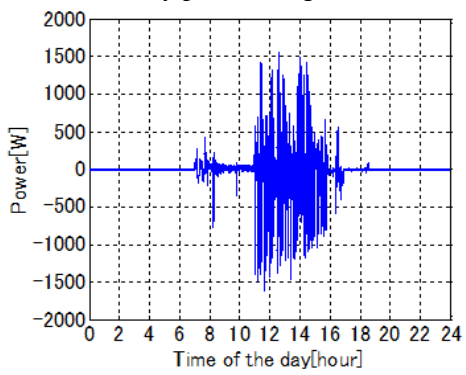

(a)

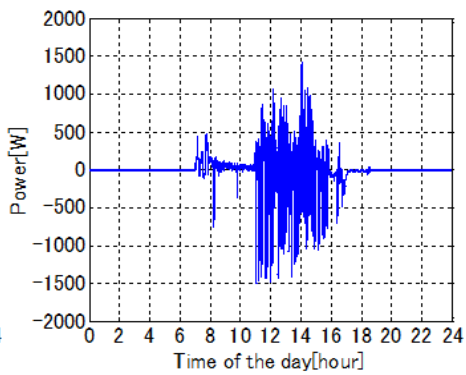

(b)
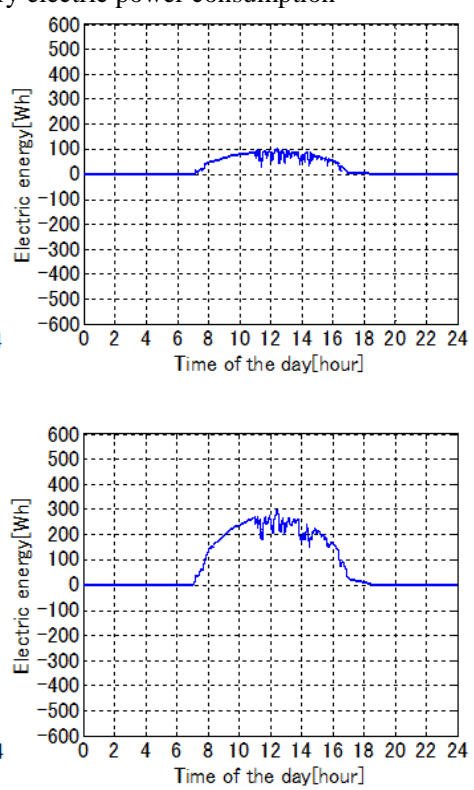



(c)

Fig. 6. PVFS simulation results using SMA method: (a) 5 minutes (Cloudy day 5/6/2012), (b) 10 minutes (Cloudy day 5/6/2012), and (c) 15 minutes (Cloudy day 5/6/2012).

Results of the Simple Moving Average (SMA) control simulation are shown in Table 1. SMA method implemented in 15 minutes shows best performance in reducing the power fluctuation for 30 minutes as understood from the PV power generation and SMA data shown in Fig. 6. However, the battery power value is increasing. A large amount of battery power influences the battery lifetime. Based on the amount of the battery power, the method of the simple moving average is most suitable for 15 minutes when the influence of battery is little. Moreover, there is little difference between the amount of the electrical charge and discharge maximum power in Simple Moving Average (SMA) method in cases of 15 minutes and 30 minutes.

Table 1. Simple Moving Average (SMA) control simulation results table

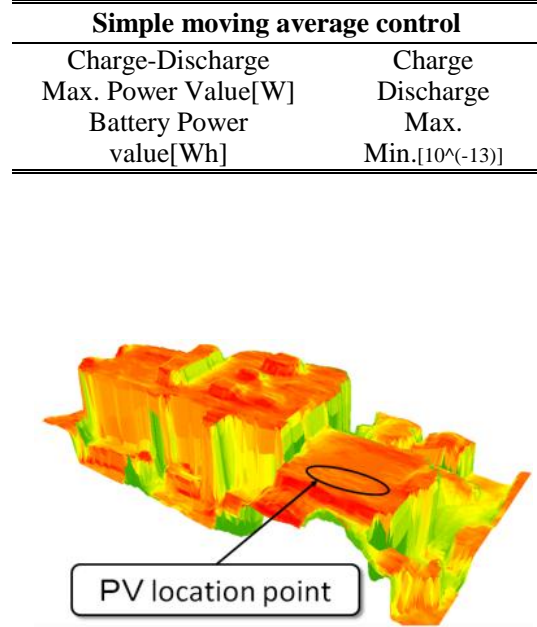

(a)

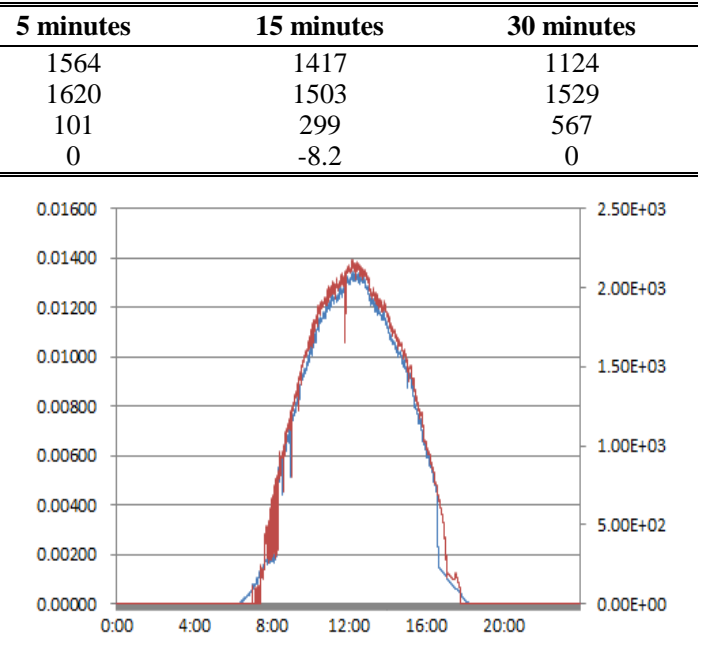

(b)

Fig. 7. GIS estimation of PV power generation at the rooftop of Building No. 10 in Kyushu Institute of Technology (Kyutech): (a) GIS location simulation of PV power and (b) Relative Comparison of Solar radiation meter and PV power output.

\section{Examination of Solar Radiation Estimation Method}

As shown in Fig. 7, steady-state power loss occurs in a sunny day in SMA control of PVFS method. In order to resolve this, estimation of solar radiation is necessary. However, such a fluctuation suppression control would tend to be used with almost all the PV power generation controls of the existing residential PCS. If sunlight energy density in the solar panel is observed, it will become possible to perform a more stable PV electric power fluctuation suppression control through a bidirectional DC/DC converter. Furthermore, calculation of the PV solar energy density in the PV installation location can be performed. As shown in Fig. 7, it is possible to construct a solar energy system in the area with best decentralized 
energy distribution as estimated by the 3D-GIS observing system.

\section{Conclusions}

In this research, PVFS method with SMA control is used in charging and discharging of electric power in batteries to suppress the fluctuations of the PV output. Based on the simulation result, it is possible to sufficiently suppress the fluctuation of PV output in each household by compensating 1.0-1.2kW. In other words, if the system is introduced into one million households in a certain area, it corresponds to a Load Frequency Control (LFC) capacity of $1 \mathrm{GW}$ of thermal power station. Also, PV change inhibitory control for weather change and SMA stable error power loss reduction becomes possible by utilizing GIS PV power estimation. The construction of a stable, economical, clean and safe energy supply system to support sustained economic and social development in line with sustained energy development has been proposed.

\section{References}

[1] Central Research Institute of Electric Power Industry (CRIEPI). (November 2010). World energy situation based on bp statistical review of world energy 2010 and other energy information. [Online]. Available: http://criepi.denken.or.jp/jp/kenkikaku/ report/ leaflet/ M10002.pdf

[2] Takaaki K, Toshiro F. Grid-connected technology and solar and wind power generation. 1st ed. Japan: Ohmsya, 2010.

[3] Masaru K, Ryota S. Resources and energy and low-carbon society. Japan: Sankyosyuppan, 2011:8.

[4] Akihiko Y. Energy New Book Smart Grid. Inc. Electric Association Newspaper club, Japan; 2011:147-150.

[5] Japan Automobile Research Institute. (2006). Recommendations for the future of next-generation vehicle batteries. study $\begin{array}{lllll}\text { group on next-generation } & \text { behicle } & \text { batteries. }\end{array}$ http://www.meti.go.jp/english/information/downloadfiles/PressRelease/ 060828VehicleBatteries.pdf

[6] Mitsutaka Y, Takao T, Tsutomu O, Takuhei H, Tadahiro G, Naoto S, Fumitoshi N, Hidemi E. A study on synchronous stability of power system with a large amount of PVs considering voltage characteristics. The Papers of Joint Technical Meeting on Power Engineering and Power Systems Engineering, the Institute of Electrical Engineers of Japan. 2011:35-42.

[7] Shinichiro N, Yasunori M, Masayuki W, Hisafumi Y, Kiyotaka F, Katsuhiko H. The isolated power system with PV supported by electric vehicle. The Papers of Joint Technical Meeting on Power Engineering and Power Systems Engineering, The Institute of Electrical Engineers of Japan. 2012:83-88.

[8] Pham XK, Yasunori M, Masayuki W, Hisafumi Y, Kiyotaka F. Development and evaluation of DC-DC converter for isolated pv power supply system with EV. The Papers of Joint Technical Meeting on Power Engineering and Power Systems Engineering, The Institute of Electrical Engineers of Japan. 2012:89-93.

[9] Pham XK, Yasunori Mi, Masayuki W, Hisafumi Y, Yaser QS, Kiyotaka F. Development and evaluation of DC-DC converter for isolated PV power supply system with EV. In: Proc. the 10th International Power and Energy Conference. 2012:242-247.

[10] Kiyotaka F, Shinichiro N, Yasunori M, Masayuki W, Hisafumi Y. Evaluation of functional isolated power supply system using PV generation and electric Vehicle. The 2013 Annual meeting of the Institute of Electrical Engineers of Japan. 2013:470-471.

[11] Kiyotaka F, Yasunori M, Masayuki W, Hisafumi Y, Yaser QS, Shinichiro N. Evaluation study of an isolated agile power system with pv and electric vehicle integration based on general model. In: Proc. 1st International Smart Grid Conference \& Exhibition. 2013:112-115.

[12] Takahisa K, Yasunori M, Masayuki W, Kiyotaka F, Yaser QS. Power supply system using electric vehicle for stable power delivery from PV Generation. In: Proc.1 st International Smart Grid Conference \& Exhibition. 2013:116-120.

[13] Atsushi S, Kiyotaka F, Takahisa K, Yasunori M. The outline of the photovoltaic simulation system using GIS. In: Proc. the 66th Joint Conference of Electrical and Electronics Engineers in Kyushu. 2013:207-208.

[14] Atsushi S, Kiyotaka F, Takahisa K, Yasunori M. System design of the photovoltaic power generation simulator using GIS Vehicle. In: Proc. theThe 2014 Annual Meeting of the Institute of Electrical Engineers of Japan. 2014:187188. 